\title{
An Active Lower Limb Orthosis Based on Electromyogram Signals
}

\author{
Yi-Jhen Li, Jian-Shiang Chen*, Chuan Yang \\ Department of Power Mechanical Engineering, National Tsing Hua University, Taiwan
}

Copyright $\bigcirc 2016$ by authors, all rights reserved. Authors agree that this article remains permanently open access under the terms of the Creative Commons Attribution License 4.0 International License

\begin{abstract}
In this article, the author aimed to design a lower limb orthosis for the person whose lower limb can emerge the normal electromyography. According to the motion intention method, use the electromyography signal and upper limb angle as the trigger signals of motion. The lower limb electromyography signal is processed by linear envelope method, and the signals are sent to the controller. The system uses PI current feedback control to give the wearer's knee appropriate aids. This orthosis is an exoskeleton with four flat EC motors as the actuators mounted on the outer of the wearer's both knees and hips, besides, uses a battery as the power source. While motor rotating, motor sends the torque to the wearer, therefore, the wearer can reduce forces applied while sitting or standing, and reach the aid purpose.
\end{abstract}

Keywords Sit to Stand, Stand to Sit, Gait Cycle Analysis, Motion Intention, Electromyogram

\section{Introduction}

As the technology grows rapidly, the development of orthosis draws more attention to people. The word "orthosis" is raised from Greek word "orthos." According to International Society for Prosthetics and Orthotics, the definition of orthosis is "externally applied device used to modify the structural and functional characteristics of the neuro muscular and skeletal systems; externally applied device used to compensate for impairments of the neuromuscular and skeletal systems." Recently, orthosis is not only for patients to fixed joints or supporting, but for normal people to strengthen force and improve the quality of life.

Some patients are suffering from the diseases which lead that lower limb muscles are not strong enough to support the daily motion. Although there is wheelchair to assist patients, but it is restricted to some terrains, like stairs [8]. A wearable orthosis is similar to human's legs, thus, it can conquer different terrains, even for normal people, they can feel tired while walking for a long distance or carrying heavy stuff. If orthosis can provide the required force in a timely manner, it is able to reduce the burden of people.

In neuromuscular or spinal nerves undamaged condition, the EMG signals can be detected on skin surface during muscle applying force. Use EMG signals to be the accordance of supporting force. For the patients whose spinal cord is damaged, their muscle cannot contract normally; their EMG signals are not suitable in this article.

The origin [1] of orthosis can be tracked back in 1890s. Nicholas Yang designed an orthosis that equipped spring on legs, which people could transfer the body weight to ground through spring while running or jumping. HAL [2] was developed by University of Tsukuba, it uses bioelectrical and joint angle as feedback signal, and uses feet pressure to judge the condition of the body weight. It is designed to enhance human force. BLEEX [3] is a lower extremity exoskeleton which is designed by University of Berkeley. It is made for the soldiers and workers in order to carry heavier and more loadings. Although BLEEX is heavy, it does not cause any burden to the wearer. The weight of BLEEX is all transferred to the ground by the structure of the mechanism. Orthosis is not only for maturity but also for medical. eLEGS and EKSO bionics [4] are two of the orthosis made for medical issues. EKSO bionics was developed in 2013, the purpose is to help patients walk dependently by themselves. It judges the motion intention according to the four point gait. The previous orthosis in our laboratory is designed by Hsu [7] and improved by Tseng [5]. It is designed for those who suffered from Degenerative arthritis. It is combined with motors, coils and M-type springs. As the knee bending, motors rotate and roll the coil, then the M-type springs bend and store elastic energy. Elastic energy is released as the assisting torque when the wearer needs. The supporting force is based on the EMG signal of rectus femoris.

\section{System Description}

\subsection{EMG Pre Processing}


Due to orthosis has to be controlled in real time, in this article, we use a designed circuit called linear envelope to get the amplitude of EMG signal. Linear envelope is one full wave rectifier plus one low-pass filter. The full wave rectifier circuit is shown in Figure 1, and the low-pass filter which is shown in Figure 2. In this article, choose the 5rd order Butterworth low pass filter and the cut off frequency is $10 \mathrm{~Hz}$.

The EMG pre progress flow chart is shown in Figure 3. The EMG signal passes through an OP amplifier to amplify the EMG signal first, and then the signal passes through a full wave rectifier circuit. After getting the full wave signal, let the signal pass through Butterworth low-pass filter, and get the linear envelope of EMG signal.

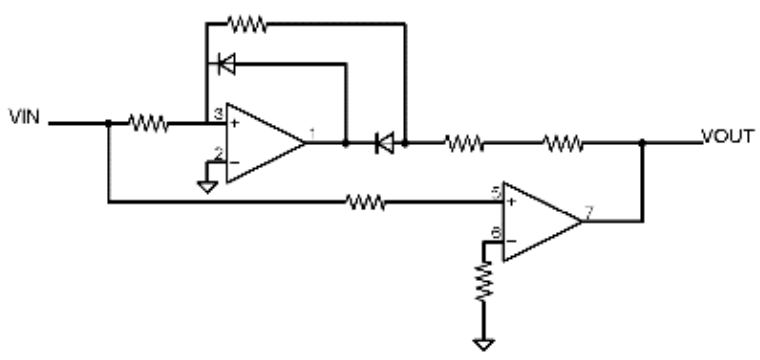

Figure 1. Full wave rectifier circuit [9]

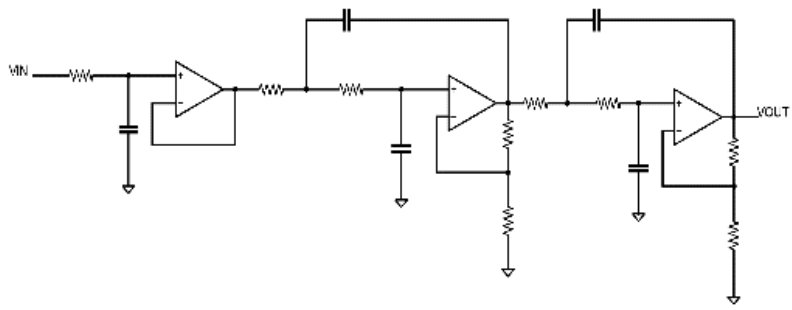

Figure 2. Butterworth low pass filter circuit [10]

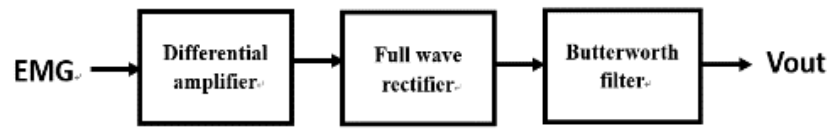

Figure 3. EMG pre progress flow chart

\section{Strategy}

\subsection{EMG Signals}

For the motion stand to sit and sit to stand, rectus femoris is the main muscle which applies force. Therefore, in this article, we choose rectus femoris as the measured muscle. Also, design a mechanism, shown in Figure 4, to find the relationship between the knee torque and electromyogram signal on rectus femoris. This mechanism is combined a torsion spring and a goniometer [13]. The measuring method is shown in Figure 5. The testing person sits on a platform, make the knee place right beside the center of the torsional spring. The testing person applies force to the mechanism and lifts up. Record the EMG signal of the rectus femoris and the data of goniometer, then calculate the spring torque and corresponding EMG signal. Repeat the experiment with difference forces, and find the relationship between the EMG signal and the knee torque.
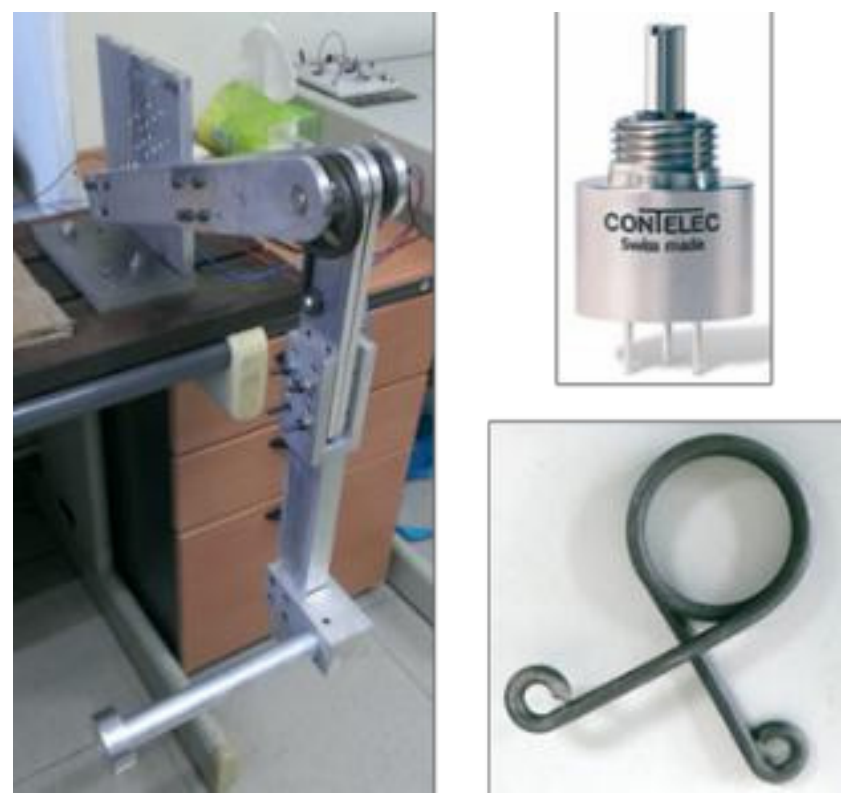

Figure 4. Knee torque test mechanism

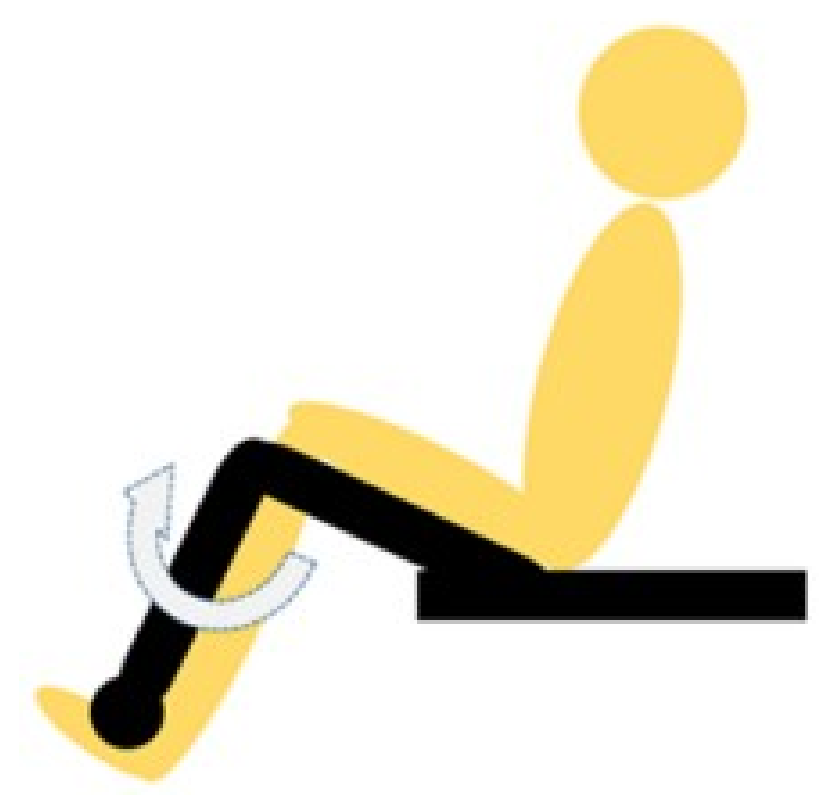

Figure 5. Knee torque measurement

The EMG signal varies from person to person. According to the experiment, assume that the relationship between the rectus femoris EMG signal and the knee torque as a linear equation, the result is shown in Figure 6.

$$
\mathrm{T}=9.1512 \mathrm{v}
$$

$\mathrm{T}$ : knee torque $(\mathrm{N} * \mathrm{~m})$ v: EMG signal $(\mathrm{V})$ 


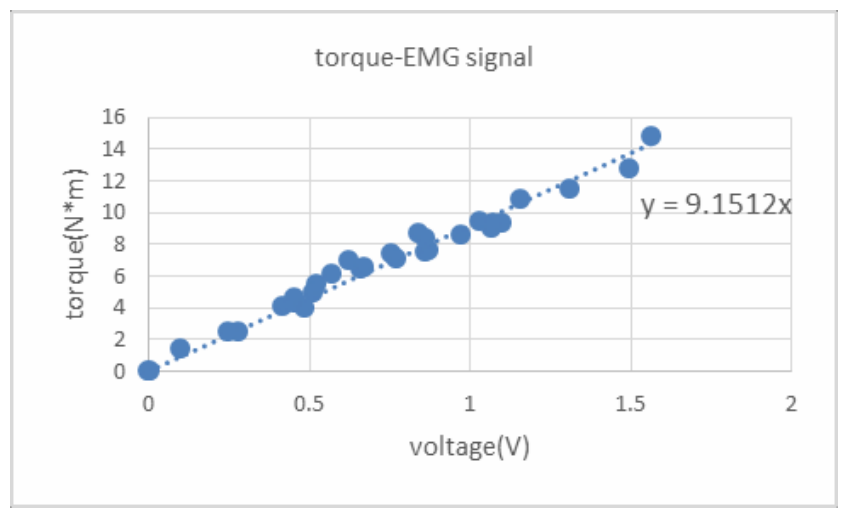

Figure 6. Relationship between knee torque and EMG signal of rectus femoris

\subsection{Weight Compensation}

According to the paper [6], for a two-link mechanism, given torque $T_{1}$ and $T_{2}$ at two joints, we can get the endpoint force F. In case, the magnitude and direction of endpoint force are known, then the joint torque can be got by calculation due to the relationship between links. In this experiment, assume $T_{1}$ as knee torque, $T_{2}$ as hip torque, and the endpoint force as F. F is the total force of compensating weight of the body and orthosis mechanism. As in Figure 7, the two-link mechanism is simplified into two sticks, as Figure 7(a), and (b) is the torque and forces separately to each link. Due to the result of torque balance, we can get the relationship equations in (2), (3).

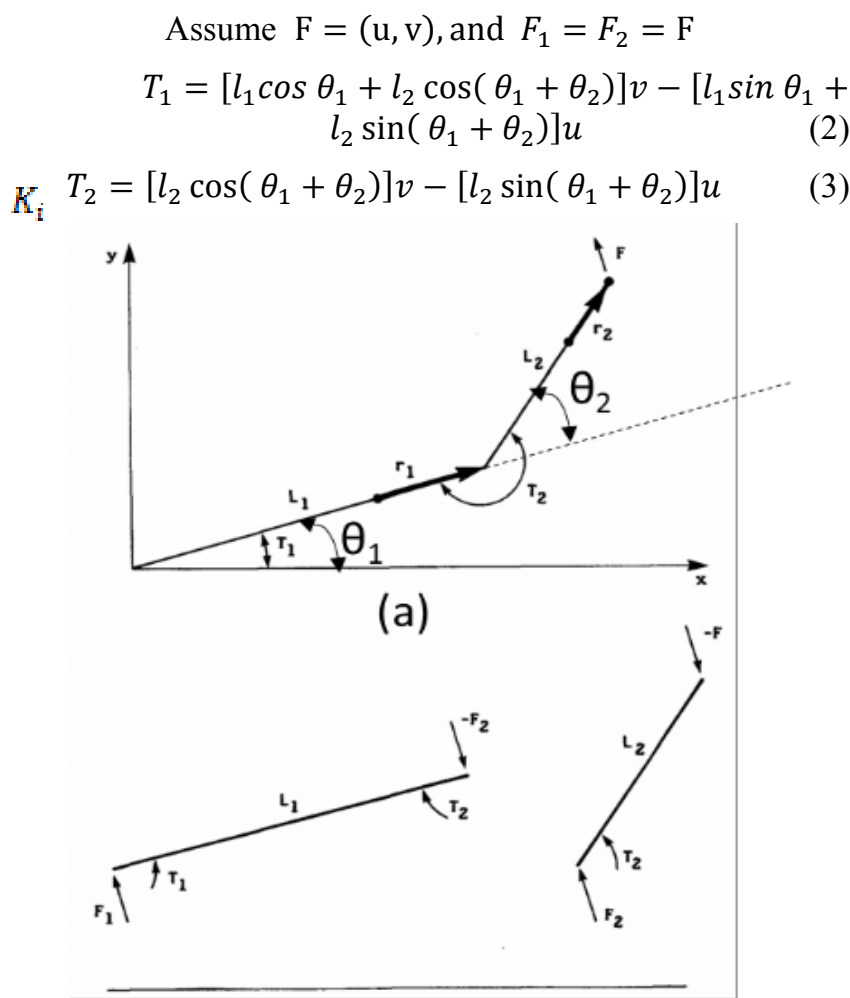

(b)

\subsection{Control Static}

Observe the daily motion of human and separate sit to stand motion into three different parts, shown in Figure 8. $\theta$ is the angle between the thigh supporting and the perpendicular direction. When $\theta$ is greater than 60 degrees, the direction of the weight compensation force and thigh supporting is in a vertical direction. When $\theta$ is between 0 degree and 60 degrees, the direction of the weight compensation force and the perpendicular direction is in 30 degrees. When $\theta$ is 0 degree, the direction of the weight compensation force and the waist support is in vertical direction.

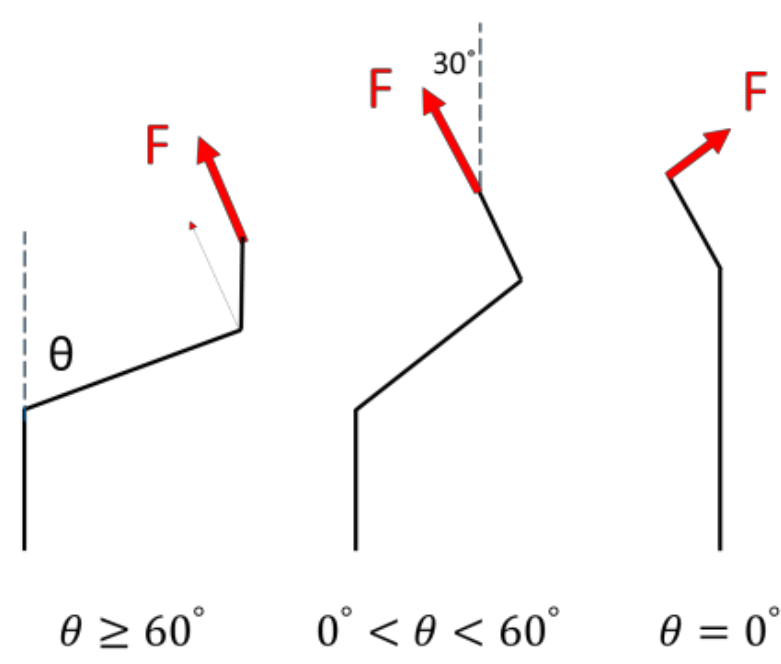

Figure 8. Sit to stand trajectory design

\subsection{Control Strategy}

The system structure is shown in Figure 9. Use the mechanism in chapter 3.1 to get the relationship between the EMG signal of rectus femoris and the knee torque. Then process the EMG signals through the linear envelope circuit in real time, compare the processed EMG signal and reference signal, and get the knee torque by the EMG signal-knee torque table. To decline the influence the weight of orthosis and the friction of motor, calculate weight compensation torque and friction compensation torque. According to the equation $T_{m}=K_{i} * i,\left(T_{m}\right.$ : motor torque, $K_{i}$ :motor torque constant, $i$ : motor current), get the current motors need by calculation, and give current command to four motors. Motors rotate and transfer torque to orthosis, and transfer to the wearer by orthosis mechanism. In this article, use PI (Proportional + Integral) current control. Choose the EMG signal when muscle is in static as reference EMG signal. Compare the EMG signal measured from the wearer with the reference signal, and do the current feedback.

Figure 7. Two links torque 


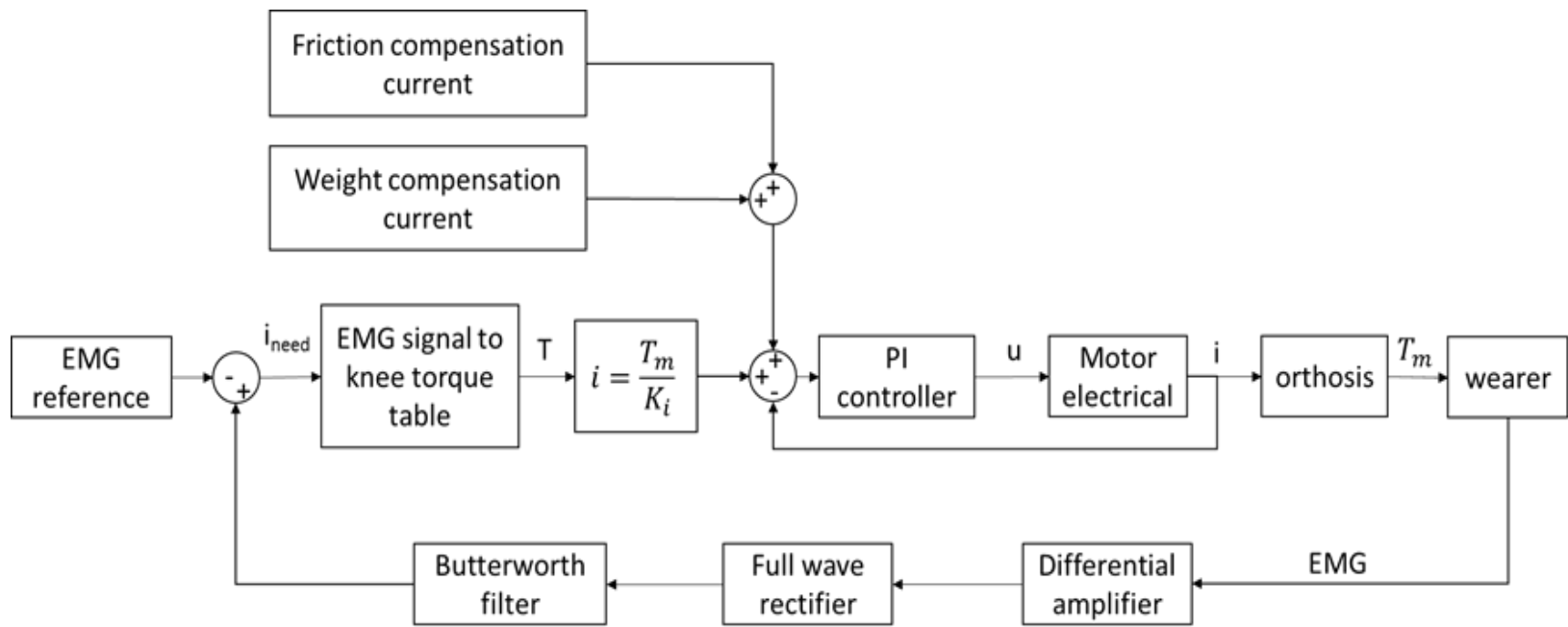

Figure 9. System flow chart

\subsection{Motion Detection [12]}

Motion estimation classify the motion gait cycle by observing human's motions in daily life. To classify different motions, put sensors on the body. According to data get from each sensor, classify the motions into different states.

Table 1. Is the motion state definition and the description of Stand to sit and Sit to stand.

\begin{tabular}{|c|c|}
\hline State & Description \\
\hline S1 & Seated on the chair \\
\hline S2 & upper limb leans with threshold angle \\
\hline S3 & stand \\
\hline T1 & upper limb leans forward \\
\hline T2 & standing \\
\hline T3 & sitting \\
\hline
\end{tabular}

\subsection{Sit to Stand}

Sit to stand motion is classified into three static states, S1, S2, S3, and two transient states, T1, T2. In sit to stand motion, initial state is seated on the chair, S1. Then the upper limb leans forward to transfer body weight, $\mathrm{T} 1$. As upper limb angle exceeds the threshold, it represents that the body weight has already transferred, and the state is defined as S2. Then starts standing movement, defines the state as T2, until finish the movement, the state is S3.

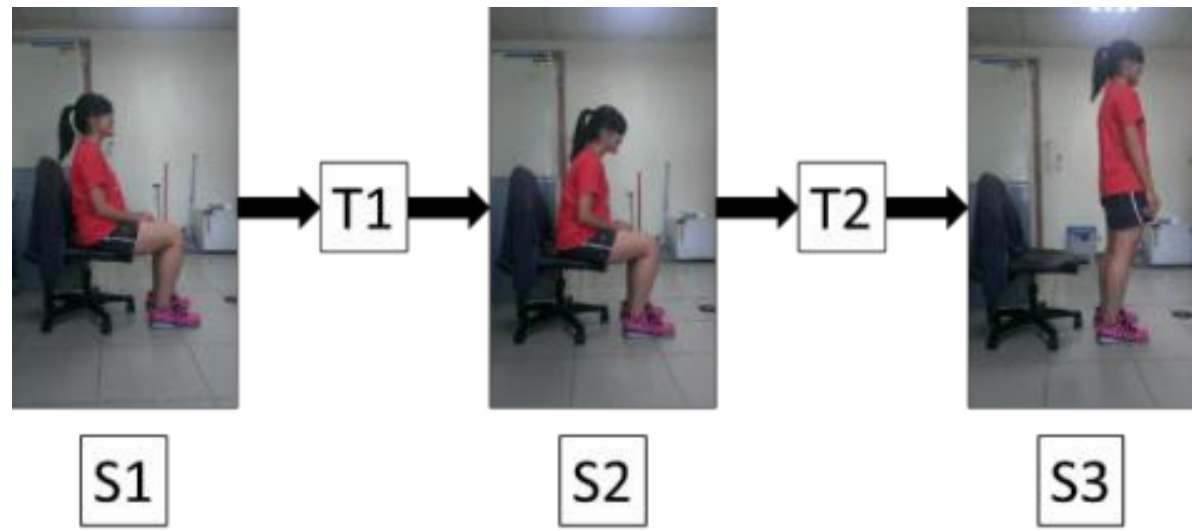

Figure 10. The motion state of Sit to Stand 

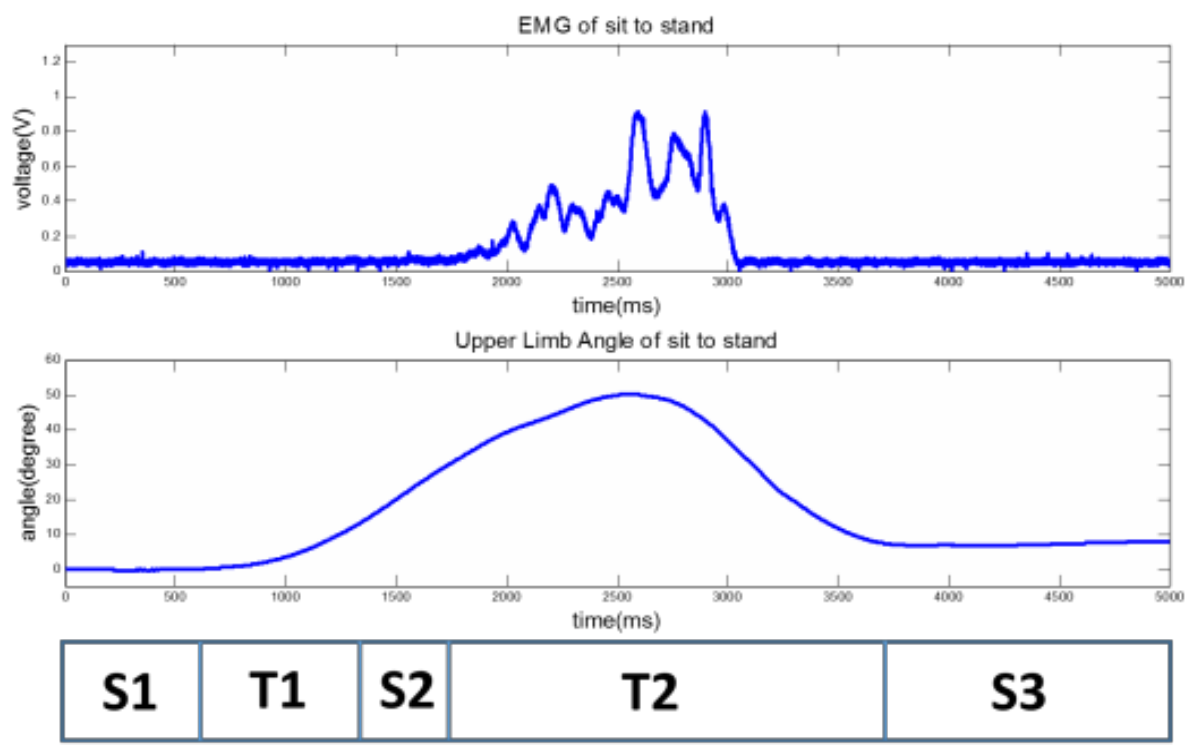

Figure 11. EMG signal and upper limb angle in sit to stand motion

\subsection{Stand to Sit}

Stand to sit motion is also classified into three static state, S1, S2, S3, and two transient state, T1, T3. The first state is stand, defines the state as S3. Before sitting on the chair, the upper limb will lean forward to transfer the body weight, which is defined as T1. In order to know whether the body weight has transferred or not, a gyroscope is set in front of the chest to calculate the upper limb angle. Set an upper limb threshold angle, if the upper limb angle exceeds the setting threshold, the motion state will be changed to S2.

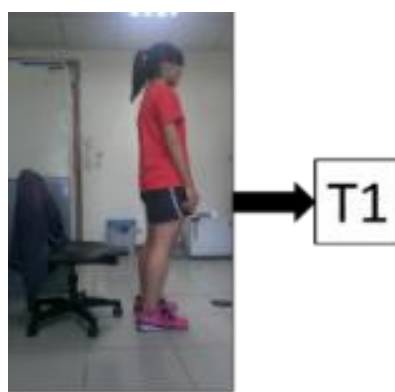

S3

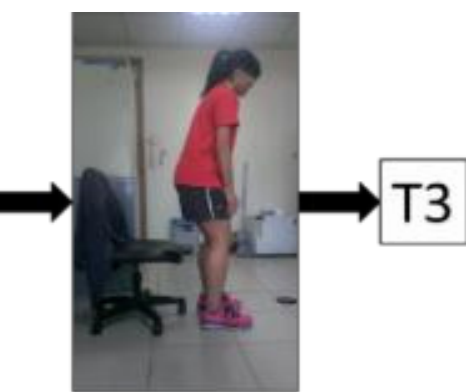

S2

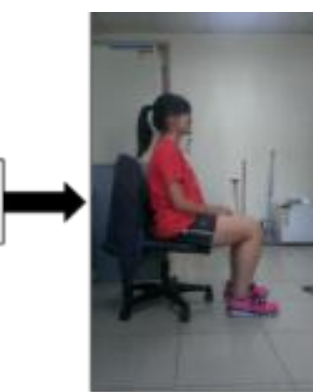

S1

Figure 12. The motion state of Stand to Sit 

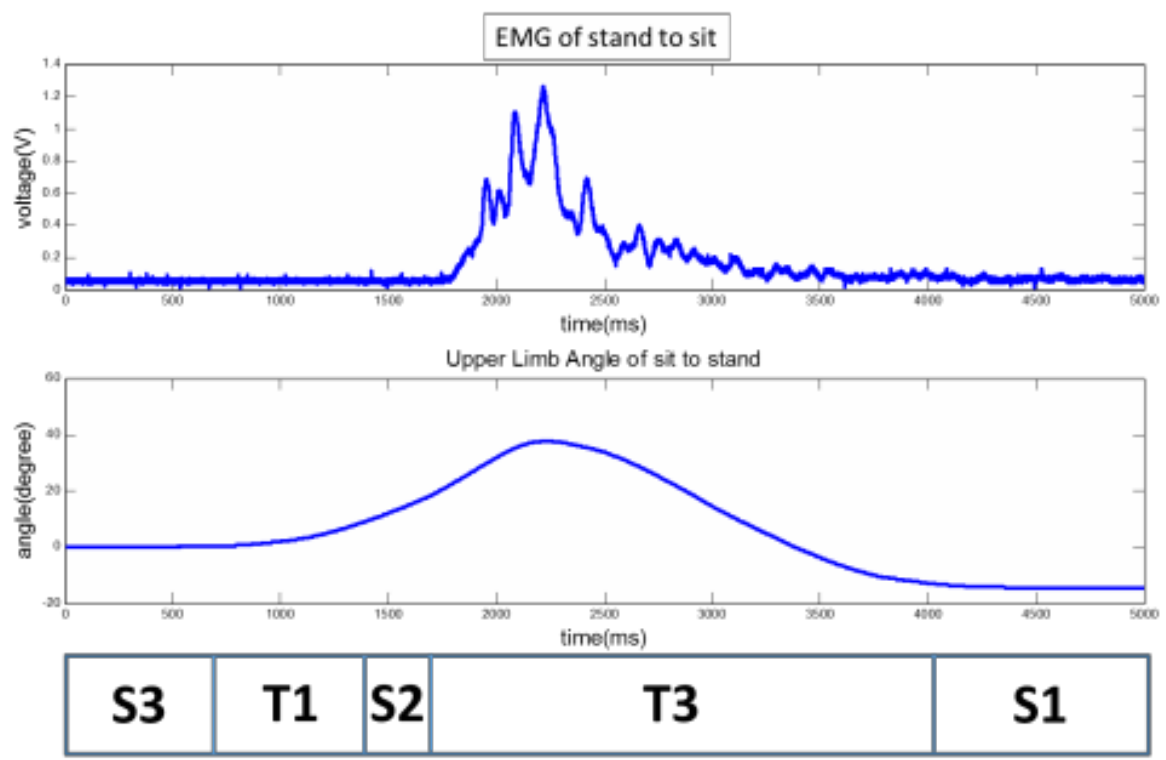

Figure 13. EMG signal and upper limb angle in Stand to Sit motion

\section{Motion Detection Flow Chart}

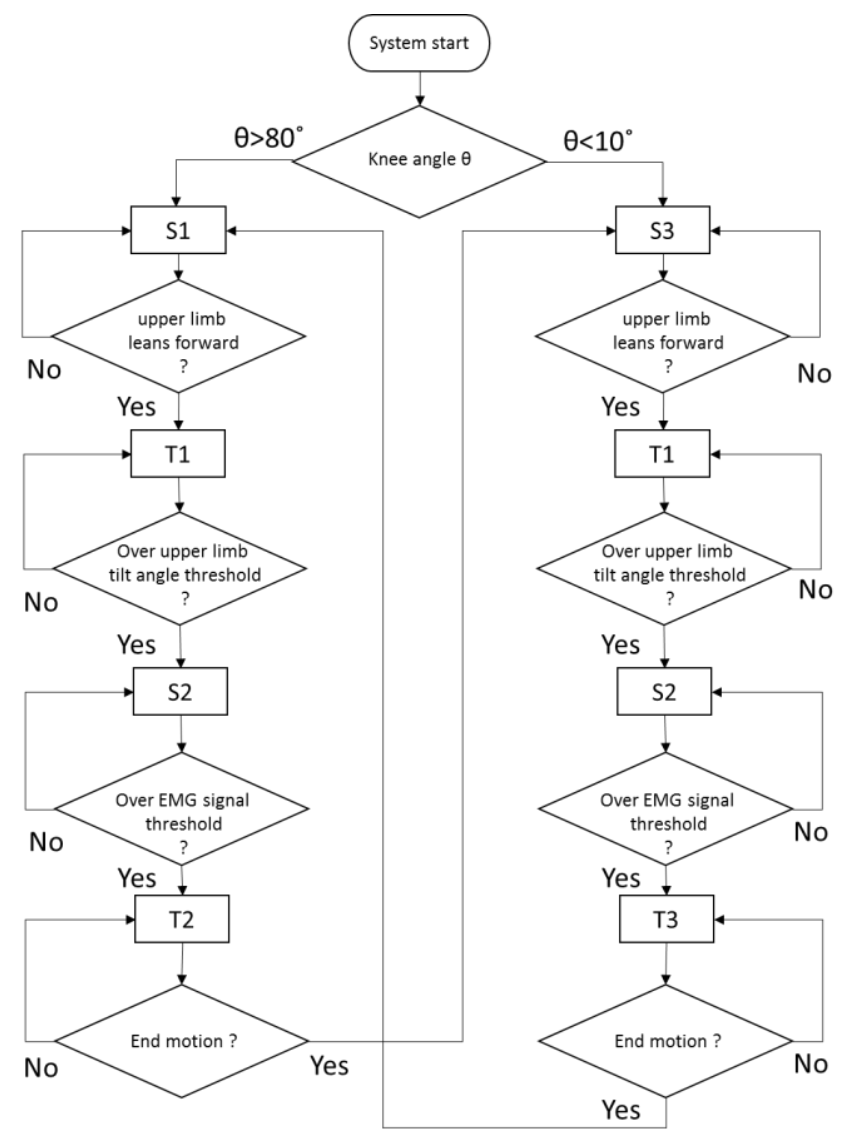

Figure 14. Motion detection flow chart

Figure 14 is the flow chart of the system. As soon as the system starts, the system judges the motion state according to the knee angle. Then determine whether the upper limb angle exceeds the threshold angle we set or not, if the upper limb angle does exceed the threshold, it means that the orthosis wearer has finished the body weight transfer, and moved into the next state. Next, determine whether the EMG signal over the EMG threshold or not. If the EMG signal surpasses the EMG threshold, it represents that the wearer has intention to sit down. Based on the initial state, if the initial state is S3, and both the upper limb angle and EMG signal are over the threshold we set, and then the state will change to T3. If the initial state is S1, and both the upper limb angle and EMG signal are also over the threshold we set, then the state will change to T2. When the system is in the transient state, T2 or T3, the motors start to operate with PI current control. After the motion finished, the state changes to the final state, and the system ends.

\subsection{System Structure}

The system structure is shown in Figure 15. There are two sensors used in the experiment. One is EMG sensor; the other is the gyroscope [11]. Collect the EMG signals through the EMG sensor, then process the EMG signal by the linear envelope circuit. At the same time, calculate the upper limb angle by the gyroscope. Capture the data to the controller EPOS2, then analysis and calculate the data. After analyzing, give the current command to motor. Motors rotate and provide torque to the wearer.

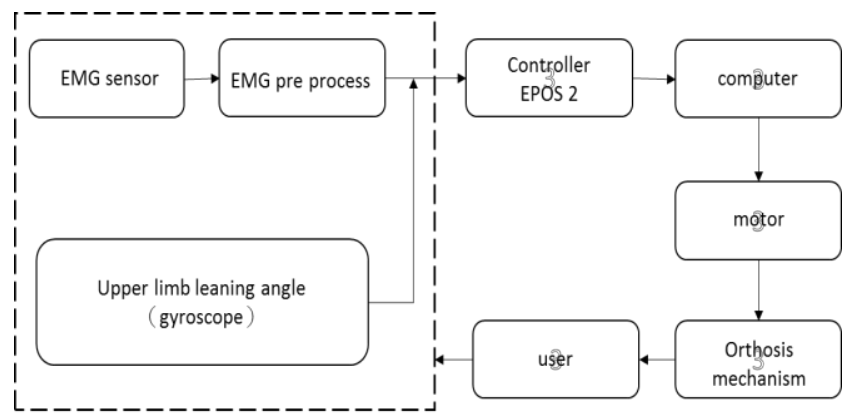

Figure 15. System structure 


\subsection{Orthosis Mechanism [14]}

The orthosis mechanism is shown in Figure 16. There are four flat brushless motors with harmonic drives. Motors are placed on the outer of both knees and hips. Four controllers are on the outside of the thigh supporting and calf supporting. Use carbon fiber as the material of thigh supporting, and steel as the waist material to strengthen the strength. Use Velcro to tie around the waist and legs, so that the orthosis can be more fit to the body. Supporting force comes from motors; each motor matches a harmonic drive, that the output torque can be increased. The weight of orthosis can be transferred to the ground through the footplate, and it will not be the burden to the wearer.

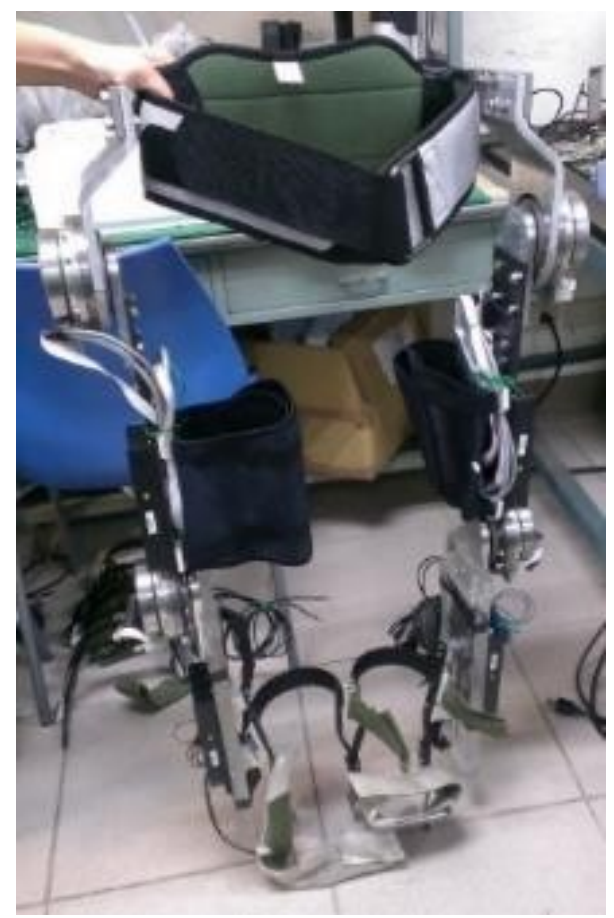

Figure 16. Orthosis mechanism

\section{Result}

\subsection{Stand to Sit}

The experiment results are shown in Figure 17 to Figure 21. Initial state is static, no current is needed. Then determine the body weight has transferred or not by upper limb angle. When it is over the threshold, it means that the body weight has transferred. If both upper limb angle and EMG exceed the setting threshold, then state will change to T3. The torque applying to the wearer is related to the current motor needs. Since the body weight and motor friction will influence the torque motor needs, which causes the current tendency applying to motor is not similar to the EMG signal. From Figure 20, we can see that when the knee angle increases, EMG signal also increases. Besides, when the knee angle increases, the knee torque caused by the body weight also increases.

The difference of EMG signal between with and without the orthosis is shown in Figure 21. The upper figure is the EMG signal without wearing the orthosis, and the figure below is the one with the orthosis. According to $\mathrm{iEMG}=$ $\sum_{n=1}^{N} x_{n}$, EMG has be reduced by $40.26 \%$, we can see that the wearer is assisted by the orthosis.
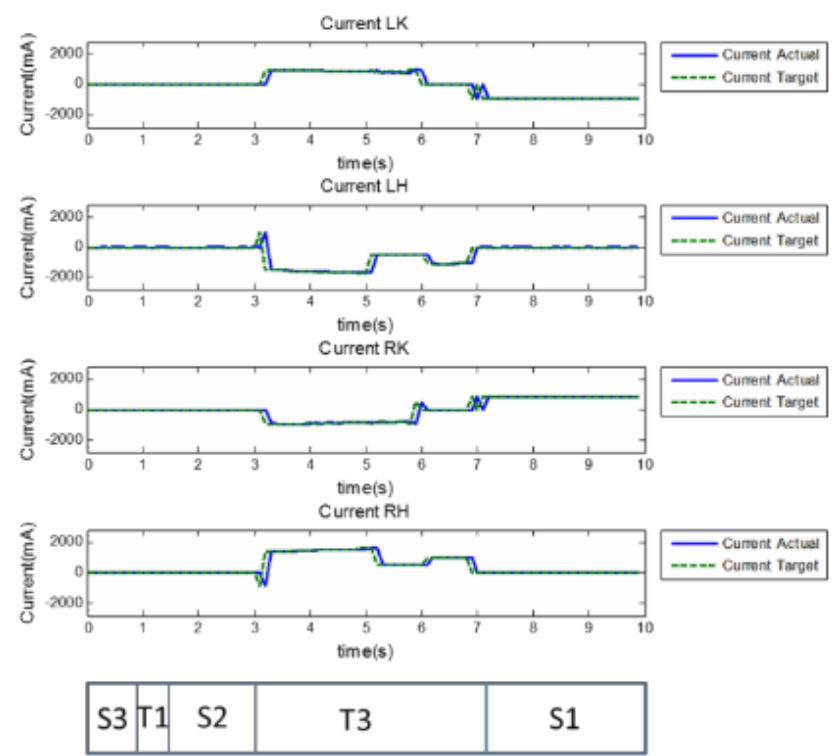

Figure 17. Motor current in stand to sit motion

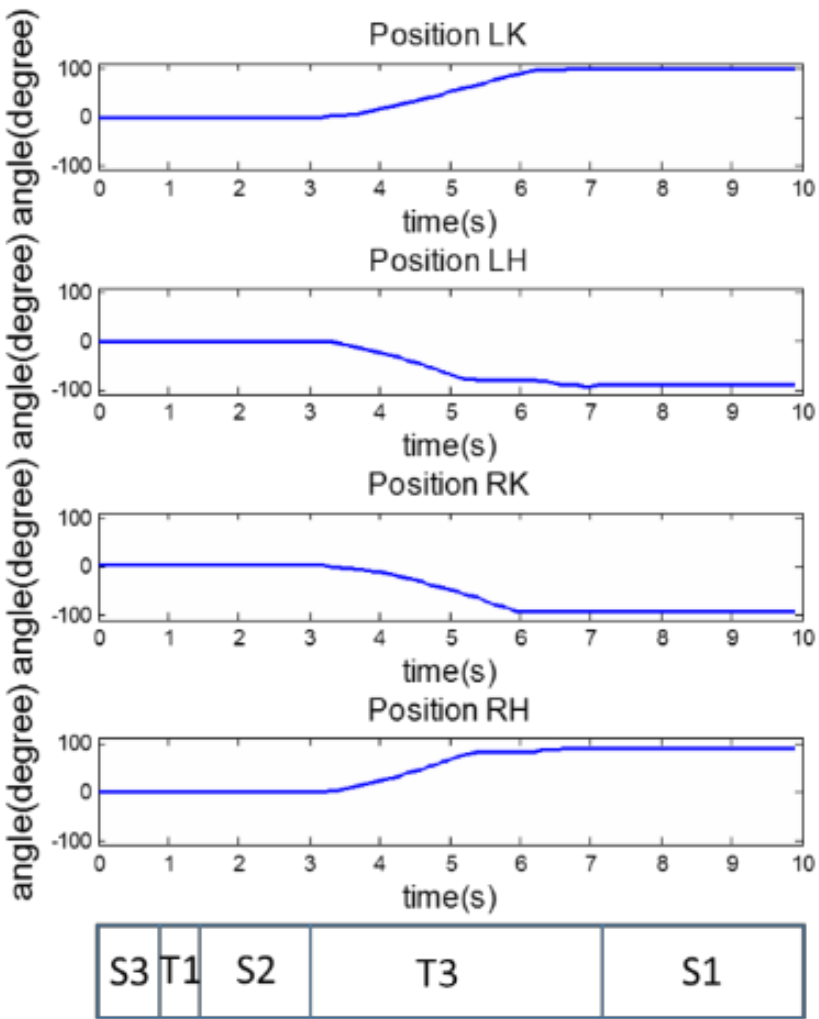

Figure 18. Motor angle in stand to sit motion 


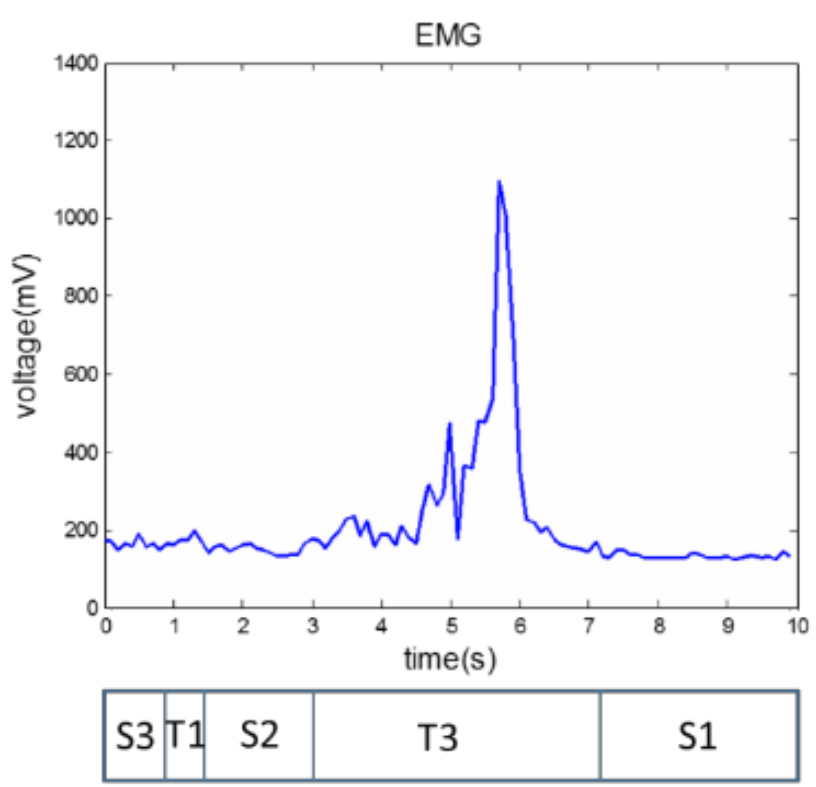

Figure 19. EMG signal in stand to sit motion

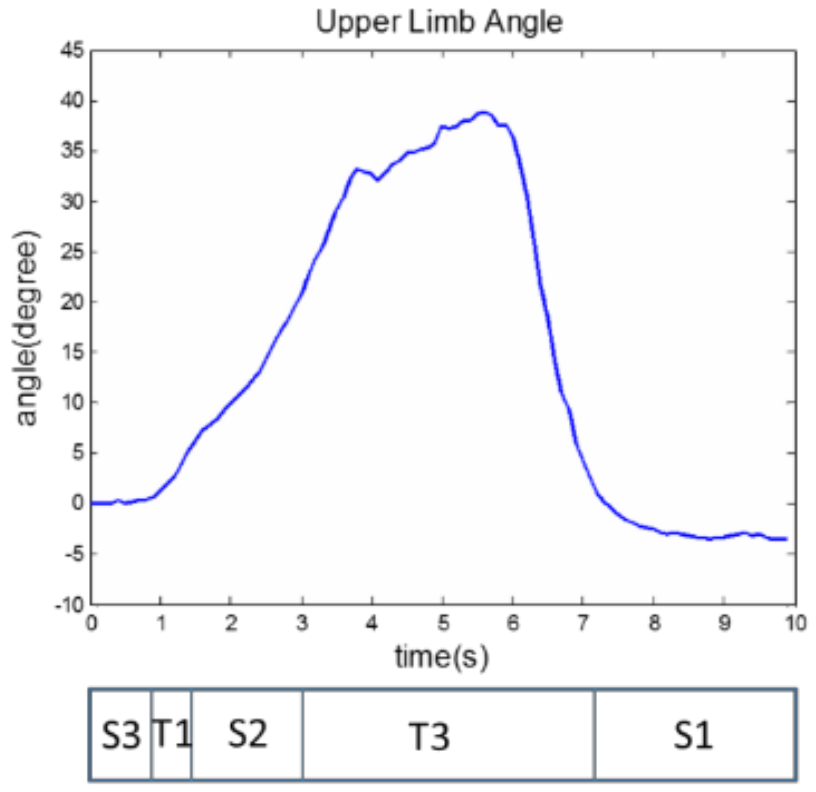

Figure 20. The upper limb angle in stand to sit motion
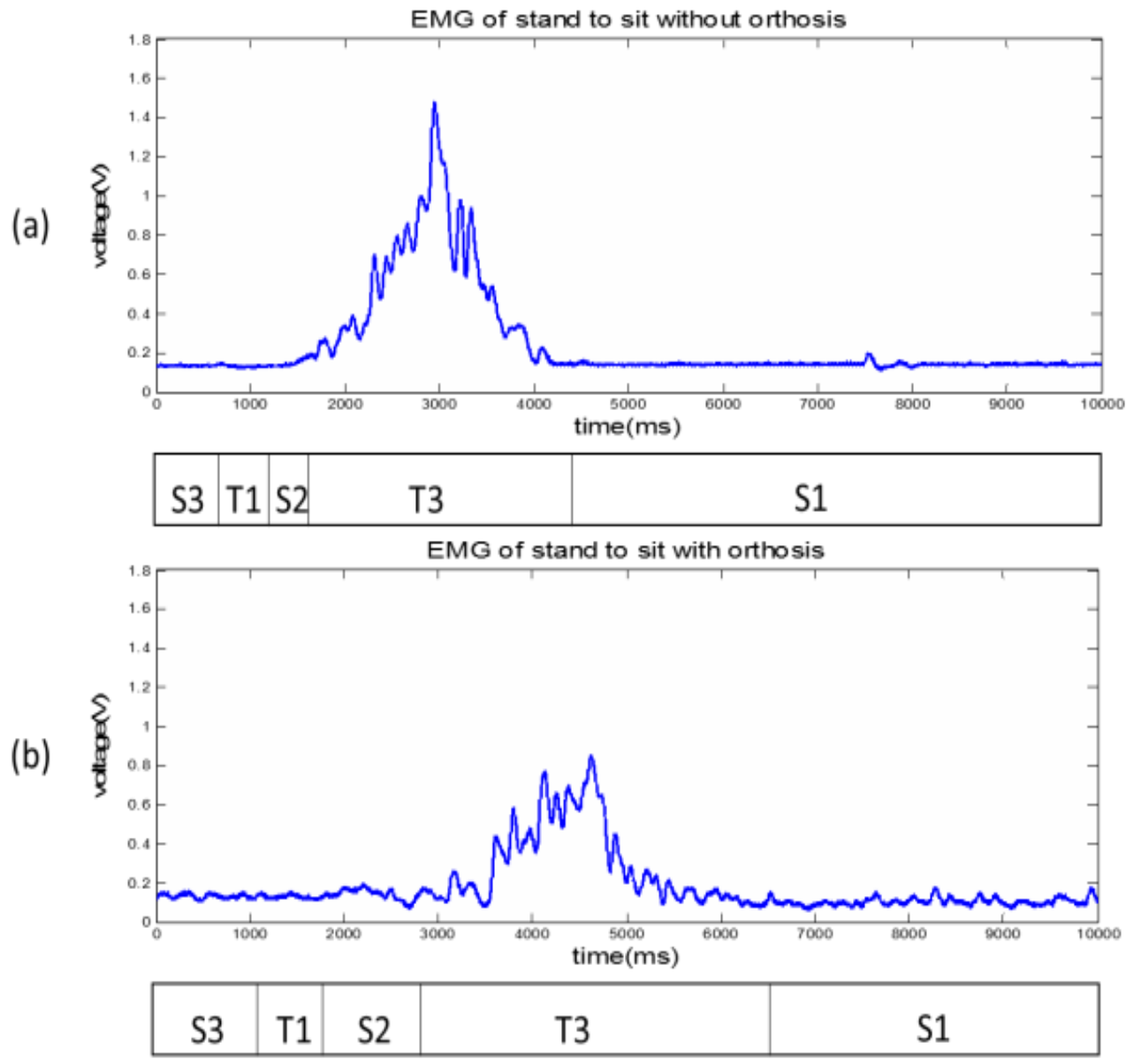

Figure 21. The comparison of the EMG signal with orthosis and without orthosis in sit to stand motion 


\subsection{Sit to Stand}

The initial state of sit to stand motion is seated on the chair, no current is needed. Calculate the upper limb angle. As the upper limb angle exceeds the threshold angle, which the body weight has already transferred. Then detect the EMG signal, when EMG signal surpasses the EMG threshold, it means the wearer has intension to stand up. When both upper limb angle and EMG signal are over the setting threshold, the motion state jump to $\mathrm{T} 2$, and the wearer starts standing movement.

According to Figure 24, the EMG signals are the greatest in the beginning of the motion. Although the body weight has transferred before wearer standing up, the wearer still needs to apply enough force to lift the body up from the chair. It is the reason why the knee torque causes by the body weight is the greatest, and the muscle needs to apply more force to support body and orthosis.

The difference of EMG signal between wearing the orthosis and without is shown in Figure 26. The figure above is the EMG signal in sit to stand motion without wearing orthosis, and the figure below is the one with the orthosis. According to the iEMG equation, the force is reduced $42.34 \%$ when the wearer is assisted by the orthosis.
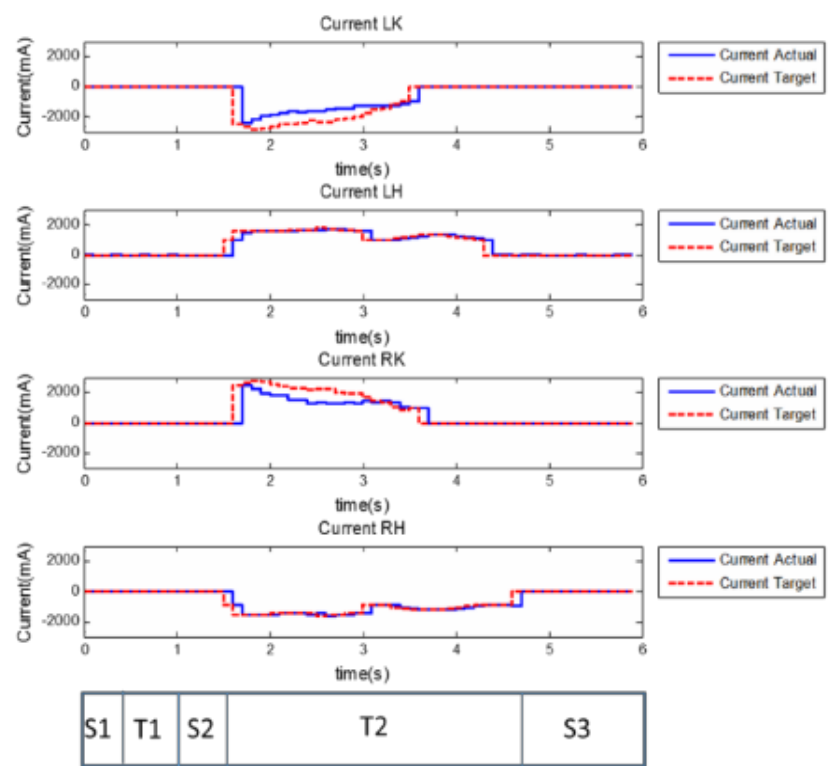

Figure 22. Motor current in sit to stand motion

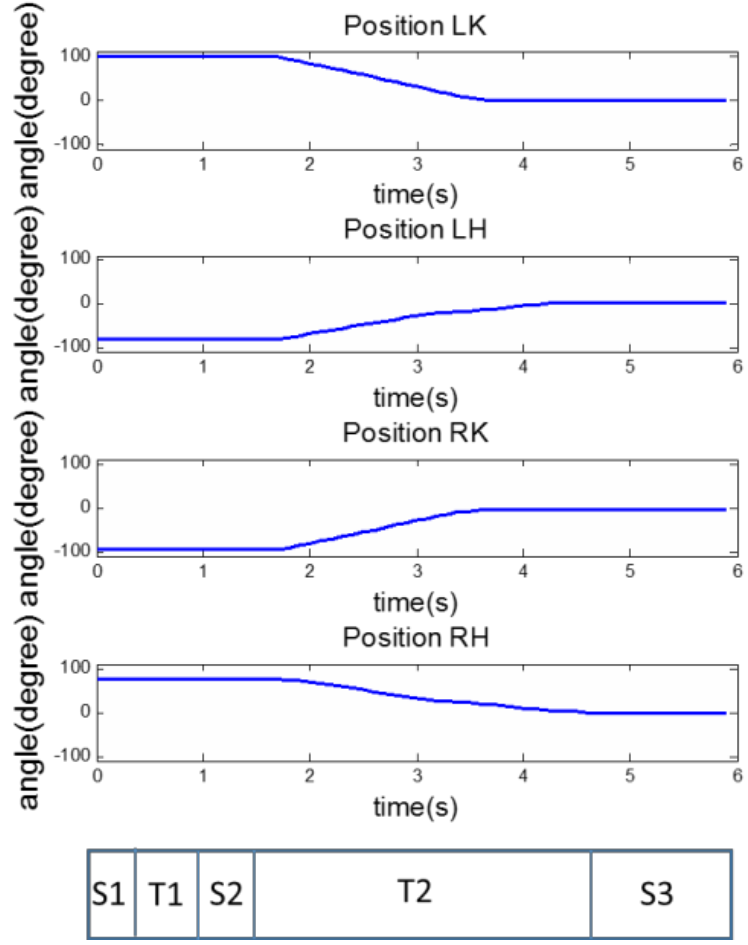

Figure 23. Motor angle in sit to stand motion

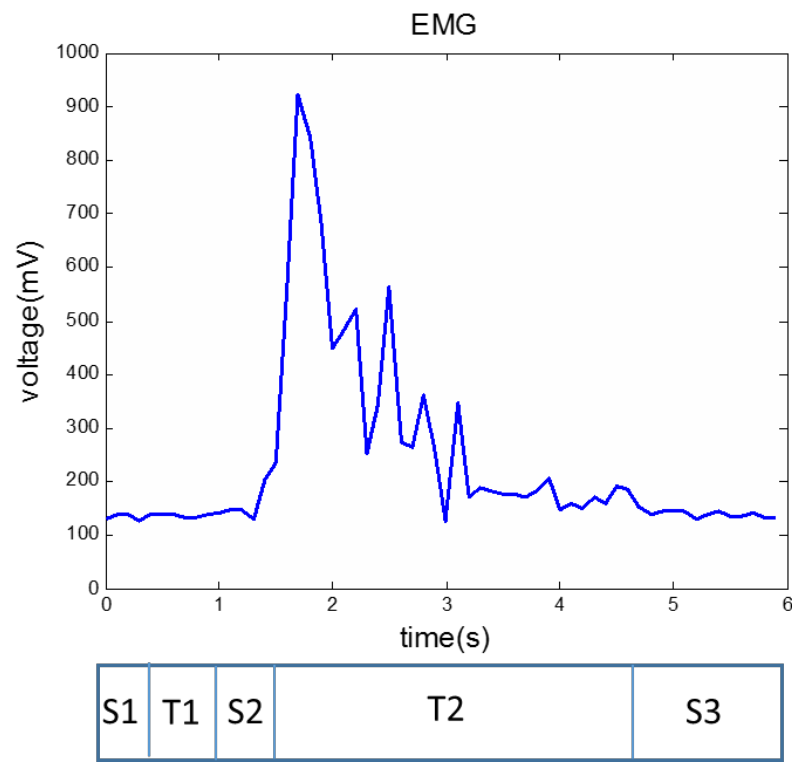

Figure 24. EMG signal in sit to stand motion 


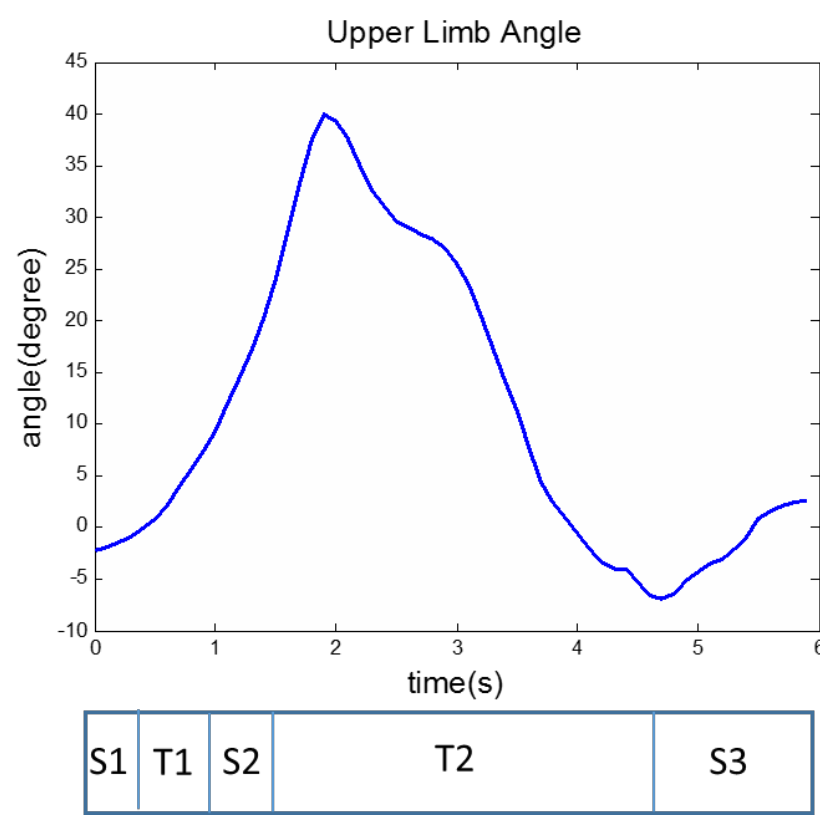

Figure 25. Upper limb angle in sit to stand motion

(a)

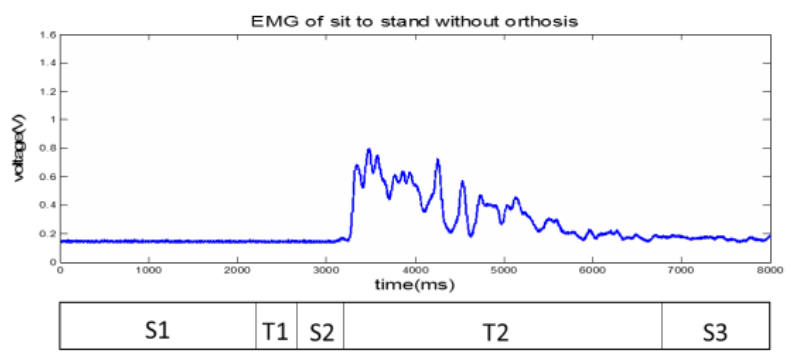

EMG of sit to stand with orthosis

(b)

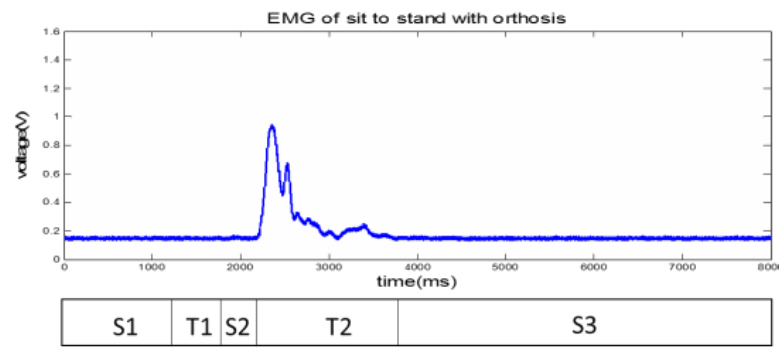

Figure 26. The comparison of the EMG signal with orthosis and without orthosis in stand to sit motion

\subsection{Walking}

The walking motion is controlled by trajectory design and current design. The gait cycle design is shown in Figure 27. Initial gait is that two legs stand in parallel, then lift up the left leg and move forward. Last, lift up the right leg and back to the origin position; First, give the left hip motor and the left knee motor current to lift the left leg and leave the ground. Then, give all motors current; make the left leg step forward and the right one push the body moving forward. Give left hip motor, right hip motor and right knee motor current, then the left leg moves back to the initial position and the right leg lifts up. Last, put the right leg down and return to the initial position. The results of walking motion are shown in Figure 28 and Figure 29.

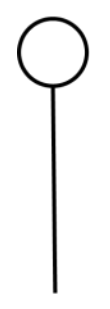

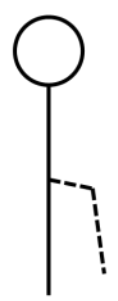
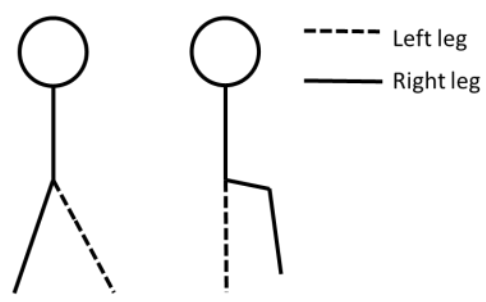

C

D
Figure 27. The trajectory of walking

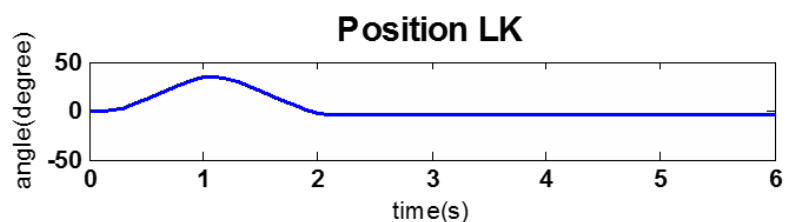

Position LH

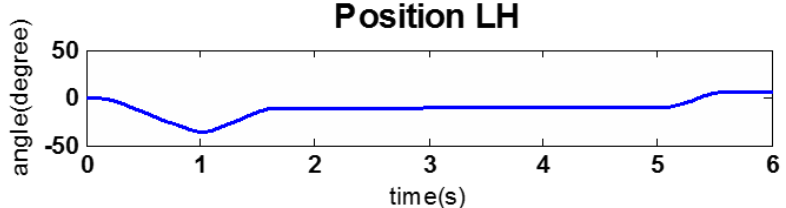

Position RK

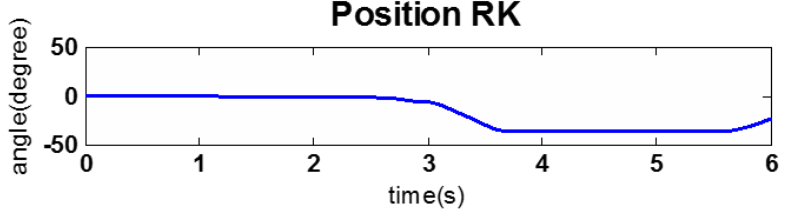

Position RH

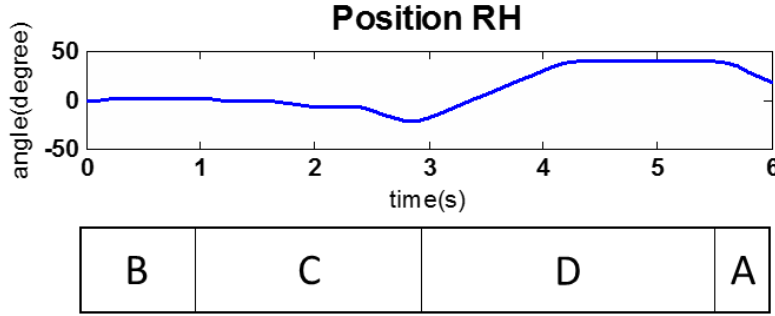

Figure 28. Motor angle in walking motion
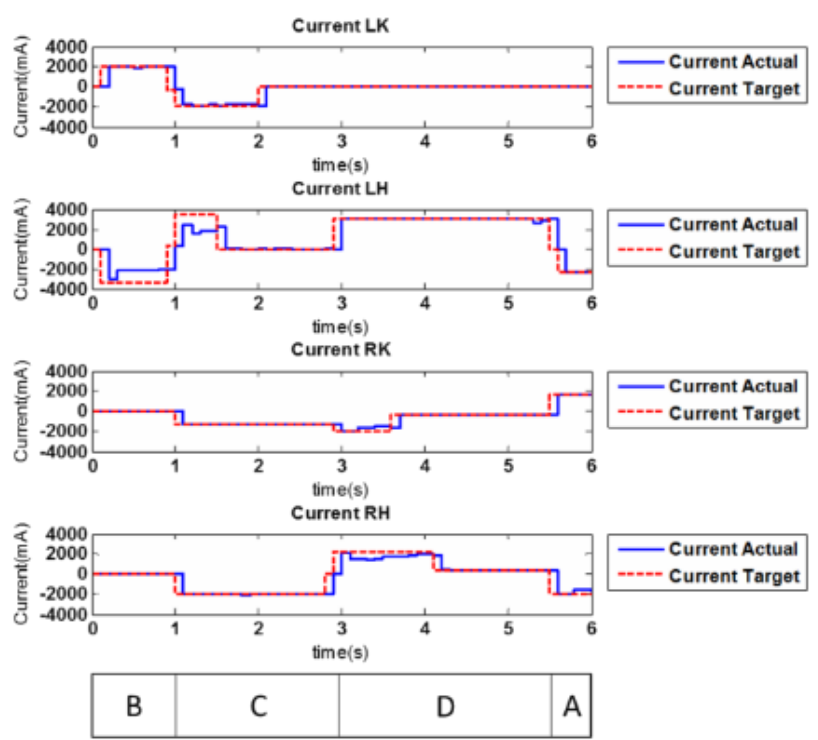

Figure29. Motor current in walking motion 


\section{Conclusions}

Orthosis provides the force to the wearer and adjusts the appropriate supporting force according to the EMG signal. Therefore, the orthosis can be more suitable for the wearer. Besides, improve the orthosis mechanism, not only reduce the weight of the orthosis but also strengthen the orthosis mechanism. In conclusion, developing an active lower limb orthosis based on electromyogram signals is worth to develop.

\section{REFERENCES}

[1] A. M. Dollar and H. Herr, "Lower Extremity Exoskeletons and Active Orthoses: Challenges and State-of-the-Art," Robotics, IEEE Transactions on, vol. 24, pp. 144-158, 2008.

[2] T. Hayashi, H. Kawamoto, and Y. Sankai, "Control method of robot suit HAL working as operator's muscle using biological and dynamical information," in Intelligent Robots and Systems, 2005. (IROS 2005). 2005 IEEE/RSJ International Conference on, 2005, pp. 3063-3068.

[3] A. Chu, H. Kazerooni, and A. Zoss, "On the Biomimetic Design of the Berkeley Lower Extremity Exoskeleton (BLEEX)," in Robotics and Automation, 2005. ICRA 2005. Proceedings of the 2005 IEEE International Conference on, 2005, pp. 4345-4352.

[4] L. Mertz, "The next generation of exoskeletons: lighter, cheaper devices are in the works," IEEE Pulse, vol. 3, pp. 56-61, Jul 2012.

[5] S.-h. Tseng, "Biofeedback of A Lower-limb Orthosis.", Mater Thesis, Department of Power Mechanical Engineering, National Tsing Hua University, Hsinchu, Taiwan, ROC.

[6] B. K. P. Horn, "Kinematics, Statics, and Dynamics of Two-D Manipulators," 1975.

[7] C.-h. Hsu, "On the Design and Implementation of Wearable Hybrid Assisted Lower Limb Orthosis", Mater Thesis, Department of Power Mechanical Engineering, National Tsing Hua University, Hsinchu, Taiwan, ROC.

[8] A. M. Dollar and H. Herr, "Lower Extremity Exoskeletons and Active Orthoses: Challenges and State-of-the-Art," Robotics, IEEE Transactions on, Vol. 24, pp. 144-158, 2008

[9] Microchip, "Microchip AN1353 OP Amp Rectifiers, Peak Detectors and Clamps datasheet."

[10] TEXAS INSTRUMENTS, "TEXAS INSTRUMENTS Active Low-Pass Filter Design Application Report."

[11] "ADXRS613 datasheet."

[12] H. A. Quintero, R. J. Farris, and M. Goldfarb, "A Method for the Autonomous Control of Lower Limb Exoskeletons for Persons with Paraplegia," ASME Journal of Medical Devices, Vol.6, Issue 4, 041003(6 pages), 2012.

[13] "PL130 datasheet", Contelec Inc., 2005.

[14] Yu, Chang-Jin, "Motion Detection for Paraplegic Patients with a Powered Lower Limb Orthosis in Ascending and Descending" National Tsing Hua University, Power of Mechanical Engineering Department Master Thesis, 2014 